\title{
Prevalence of human immunodeficiency virus - hepatitis B virus co-infection amongst adult patients in Mahalapye, Ngami, Serowe, Botswana: a descriptive cross-sectional study
}

\author{
Azhani Mandiwana ${ }^{a *}$ (D) and Stephane Tshitenge ${ }^{b}$ iD \\ ${ }^{a}$ Botswana Defence Force, Gaborone, Botswana \\ ${ }^{b}$ Family Medicine and Public Health, University of Botswana, Gaborone, Botswana \\ *Corresponding author, email:azh_mand@yahoo.com
}

Background: About 37 million people are living with human-immunodeficiency-virus (HIV) worldwide, with 2.6 million co-infected with the hepatitis B virus (HBV). HBV infection causes 650000 deaths annually worldwide. Botswana has a high prevalence of HIV and a growing population of patients on highly active antiretroviral therapy (HAART). This study aimed to determine the prevalence of HIV-HBV co-infection amongst HAART eligible adult patients in some rural settings in Botswana. Methods: A cross-sectional study was conducted amongst HAART eligible adult patients at 15 HAART clinics in the Mahalapye, Ngami and Serowe Health Districts of Botswana, from August to October 2015. A total of 132 were recruited; of these 118 consented and were tested for HBsAg reactivity using Elisa.

Results: Six $(5.1 \%, 6 / 118)$ patients from the three rural health districts were HIV-HBV co-infected, with three in the 20-29 age group. The association between sex and HIV-HBV co-infection status was not statistically significant; $p=1.00$.

Conclusion: The finding of 5.1\% HIV-HBV co-infection prevalence in some rural settings of Botswana was similar to results from one study conducted in a Botswana urban centre, while another previous similar study reported prevalence as being twice as high. This finding may call for prioritisation of pre-HAART HBV screening and early HAART initiation for all HIV-infected patients.

Keywords: Botswana, HBsAg screening, HIV-HBV co-infection prevalence

\section{Introduction}

The World Health Organization (WHO) has developed the 'three interlinked Global Health Sector Strategies (GHSS)' to address the three major public health issues, namely human immunodeficiency virus (HIV), viral hepatitis (VH) and sexually transmitted infections (STI); the institution aims to reduce twothirds of new HIV infections by 2020 and to reduce $90 \%$ of new cases of chronic hepatitis by $2030 .{ }^{1}$ Worldwide, about 36.9 million people live with HIV; of these 2.6 million are co-infected with hepatitis B virus (HBV). ${ }^{2,3}$ Primary modes of the HIV-HBV coinfection transmission include sexual contact, injection drug use, mother-to-child transmission (MTCT) and men who have sex with men (MSM), with MTCT being the major mode of transmission in high prevalence regions such as sub-Saharan Africa and east Asia. ${ }^{3,4}$

Sub-Saharan Africa accounts for about $75 \%$ of the worldwide HIV cases and has about $14 \%$ of the worldwide chronic hepatitis $B$ cases. ${ }^{2,5}$ A systematic review that covered 60 sub-Saharan Africa studies from 1990 to 2009 estimated that the HIV-HBV coinfection prevalence was $14.9 \% .^{6}$

In Southern Africa, a study conducted among South African and Zambian patients in 2008 reported an HIV-HBV co-infection rate of 7.4\%; another study conducted amongst South African and Botswana patients in 2013 reported almost the same HIV-HBV co-infection rate of $7.0 \%$.,8 Two HIV-HBV co-infection studies previously conducted in tertiary health facilities in urban settings in Botswana estimated HIV-HBV co-infection prevalences of $10.6 \%$ and $5.3 \% .{ }^{9,10}$
The endemicity of both HIV and HBV in sub-Saharan Africa raises the implications of this co-infection for the long-term health status of HIV-infected adult patients in Botswana, which has a high prevalence of HIV infection as well as an increasing population of patients on HAART. ${ }^{11}$ Knowledge of the HBV infection status of patients being initiated on HAART could facilitate individualised patient management as regards HAART drugs or regimen switches that are provider-initiated such as due to treatment failure. The prevalence of HIV-HBV co-infection in rural settings among primary care patients in Botswana has yet to be established; there is a need to know the national prevalence data as this could facilitate the implementation of the 'WHO global health sector strategy on viral hepatitis 2016-2021' in Botswana. ${ }^{2}$

This study sought to ascertain the prevalence of HBV infection among HIV-infected adult patients who meet the 2012 Botswana national HAART eligibility criteria in rural settings, in Botswana. ${ }^{12}$

\section{Methods}

A cross-sectional study was conducted among HIV-infected adult patients ( 18 years and above) who were eligible for HAART initiation, on account of CD4 counts less than $350 / \mathrm{ml}$, WHO clinical stages III or IV, or being pregnant as inclusion criteria. ${ }^{12}$ Patients who could not be screened for HBsAg, for whatever reason, were excluded from the study. Common reasons in this regard encompassed patients' decision not to participate in the study and inability to consent before providing blood specimens for pre-HAART baseline assessment. 
We selected three rural health districts, which were the Mahalapye health district, the Ngami health district and the Serowe health district, based on their proximity to the University of Botswana - Family Medicine training sites. Due to logistic constraints, we randomly selected 15 HAART clinics by picking their names from a box. The following sites were selected for this study: (i) Mahalapye District Hospital based HAART clinic (Mahalapye Health District), (ii) Maun, Sedie, Moeti, Boseja, Shorobe, Tsau, Sehitwa, Kareng, Phuduhudu and Makalamabedi HAART clinics (Ngami Health District) and (iii) Kadimo, Newtown, Nutrition and Serowe HAART clinics (Serowe Health District).

The Mahalapye Health District contributed 256 patients (36\%), Ngami Health District 249 patients (34\%) and lastly the Serowe Health District 213 patients (30\%) to the overall study population of 718. To calculate the sample size, we requested the three health districts to provide sex-specific data on their number of HAART eligible adult patients over a period of three months, from April to June 2014. They reported data on 475 female and 243 male patients, hence a sex ratio of 2:1. We used the StatCal tool of Epi Info version $7^{\circledR}$ (CDC, Atlanta,GA, USA), with a study population of 718 , expected HIV-HBV co-infection prevalence of $10 \%,{ }^{3}$ and confidence limits of 5\%, to determine a minimum sample size of 116 .

We stratified the sample sizes per health district and sex ratio. Therefore, the Mahalapye Health District recruited 42 participants, the Ngami Health District 41 participants and the Serowe Health District 35 participants, to produce an overall sample size of 118 . We maintained the sex ratio of 2:1 for female versus male in the sample size. The first patient to be recruited per sex stream per study site was pre-determined by the toss of a dice. Every sixth eligible patient (718 divided by 116$)$ per sex stream per study site was recruited for this study. In the event that the patient eligible for recruitment did not consent, consecutive patients were recruited until one consented, whereupon recruitment reverted to the interval format.

We trained 48 nurses and laboratory technicians as research assistants in 18 ( 15 clinics and 3 laboratories) study sites on the research procedures and processes. The nurses' roles were to recruit participants during HAART initiation, seek their consent and collect blood for processing by the laboratory. The laboratory technicians separated the sera and coordinated the transfer of specimens to the Botswana Harvard HIV Reference Laboratory (BHHRL).

Consent forms had pre-allocated barcode stickers with participantspecific numbers appended to them, and this facilitated linkage of the specimen and test result to a particular participant. Consent form keys were completed immediately after the participant signed the consent form; this form captured information such as the participant's name and age and the barcode number, which was identical to that appended to that particular participant's completed consent form. The consent form keys helped to link the laboratory results to individual participants.

During blood collection for the baseline pre-HAART investigations, the nurse collected an additional $3 \mathrm{ml}$ of blood in a separate plain tube and completed a laboratory requisition form for $\mathrm{HBsAg}$ testing bearing the same barcode sticker as that in the participant's completed consent form. The nurse also appended the same barcode sticker to the participant's blood specimen at the clinic before transferring it to the district hospital's laboratory in a cooler box. The district hospital's laboratory transferred the serum into a cryovial bearing the same barcode sticker; the serum, laboratory requisition form and the laboratory checklist form were then sent to the BHHRL for storage prior to analysis. A cold chain was maintained throughout this process.

The BHHRL used the HBV Murex version 3.0 Elisa ${ }^{\circledR}$ test kit (Murex Biotech Ltd, Temple Hill, Dartford DA1 5LR, United Kingdom Ref: 9F80-01) to test the samples for hepatitis B surface antigen (HBsAg) reactivity, then documented the participant's HBsAg test result on the appropriate laboratory requisition form, and thereafter sent it to the appropriate HAART clinic for filing in the participant's medical records.

Telephone communication was utilised between the district hospital laboratories and the BHHRL so as to minimise specimen rejection rate and misplacement. BHHRL has regular quality assurance assessments; it calibrated the Elisa analyser prior to running of the specimen and also conducted confirmatory repeat runs for any specimen reported as positive.

We used a register with tables for data collection to capture variables, namely: health district, health facility, age, sex and $\mathrm{HBsAg}$ results. The HBsAg test's positive result was used as the surrogate for presence of chronic HBV infection. ${ }^{13}$ We summarised our results with frequency tables and bar charts. Descriptive statistical analysis was performed using SPSS ${ }^{\circledR}$ version 21 (IBM Corp, Armonk, NY, USA). We used Fisher's exact test to assess whether there was an association between sex and HIV-HBV coinfection status. The level of statistical significance was taken as below 0.05 .

\section{Ethical consideration}

Ethical clearance was obtained from the University of Botswana Independent Review Board (ref: URB/RES/COMN/093) and the Ministry of Health of the Republic of Botswana (ref: PPME: 13/18/I Vol. IX (429). All participants provided signed informed consent. No patient identifiers were captured in the data collection sheet. Confidentiality and anonymity were ensured throughout this study, unless stated otherwise. The nurse reminded participants that they were free to withdraw their consent at any time. Participants' HBsAg results were disclosed to their clinic staff for post-test counselling and appropriate management.

\section{Results}

\section{Baseline characteristics}

A total of 132 adult patients eligible for this study were recruited, and 14 declined to participate, leaving 118 who consented and participated. Two-thirds $(80,67.8 \%)$ were female (Table 1). The participants' ages ranged from 19 to 69 , and the mean age was 35 years (standard deviation $[S D] \pm 10$ ). We observed that participants below 40 years old were likely to be female. After the age of 40 years there was parity in number of participants between the sexes, while old participants (above 60 years) were all male. Most participants were in the age groups $20-29$ years $(36,31 \%)$ and $30-39$ years $(46,39 \%)$, followed by a progressively declining number of participants per age group with increasing age (Figure 1).

\section{Prevalence of HIV-HBV co-infection}

Among these 118 participants, 6 were HIV-HBV co-infected (5.1\% [6/118], 95\% Cl 1.13-9.07\%); 4 were female $(5.0 \%, 95 \% \mathrm{Cl}$ $0.22-9.47 \%)$ and 2 were male $(5.3 \%, 95 \% \mathrm{Cl} 1.82-12.42 \%)$. The association between sex and HIV-HBV co-infection status was considered not to be statistically significant; $p=1.00$. Half ( 3 out of 6) of the co-infected participants were from the age group 20-29 years-old (see Figure 1). HIV-HBV co-infected participants had a mean age of 32.3 years (SD \pm 8.5 ) compared with the not 
Table 1: Baseline characteristics of HAART-eligible adult patients in the Mahalapye, Ngami and Serowe Health Districts, August to October 2015

\begin{tabular}{|c|c|c|c|}
\hline \multirow{2}{*}{$\begin{array}{l}\text { Patient } \\
\text { characteristics }\end{array}$} & \multicolumn{3}{|c|}{$\begin{array}{l}\text { Number of HAART eligible adult patients (study } \\
\text { participants) }(n=118)\end{array}$} \\
\hline & Males $(n=38)$ & $\begin{array}{l}\text { Females } \\
(n=80)\end{array}$ & Total $(n=118)$ \\
\hline$<20$ & 0 & 2 & 2 \\
\hline $20-29$ & 10 & 26 & 36 \\
\hline 30-39 & 11 & 35 & 46 \\
\hline $40-49$ & 10 & 13 & 23 \\
\hline $50-59$ & 4 & 4 & 8 \\
\hline $60-69$ & 3 & 0 & 3 \\
\hline Total & 38 & 80 & 118 \\
\hline
\end{tabular}

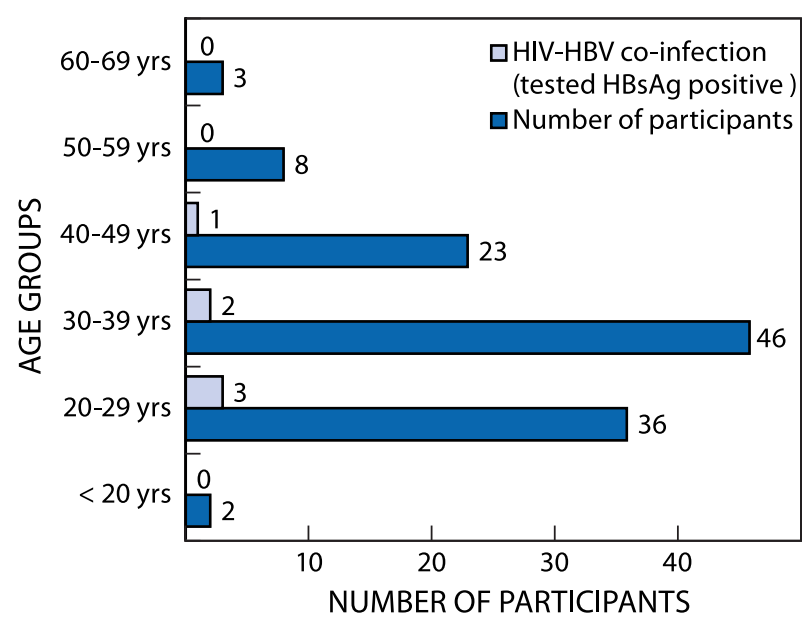

Figure 1: Bar chart demonstrating age groups of the study participants and co-infected participants from the three rural sites, Mahalapye, Ngami and Serowe, Botswana, August to October 2015.

co-infected participants who had a mean age of 35.5 years (SD \pm 10.2). Three HIV-HBV co-infected participants were from the Serowe Health District (Table 2).

\section{Discussion}

The prevalence of HIV-HBV co-infection in this study of 5.1\% (95\% Cl 1.13-9.07\%) was similar to that reported in a 2010 Botswana HIV-HBV study (5.3\%), wherein the female patients' co-infection rate was $5.4 \%$ and that for male patients was $4.7 \%,{ }^{10}$ and the Southern African region (7.0\% and 7.4\%). ${ }^{7.8}$ With the non-difference of HIV-HBV co-infection prevalence between the 2010 Botswana HIV-HBV study and our study (2015), one could deduce that the introduction of the tenofovir (TDF) and emitricitabine (FTC) mainstay HAART regimen in 2008 could have helped to stabilise the prevalence in Botswana. ${ }^{10}$ The wide confidence intervals of our co-infection prevalence results may be due to the small number of co-infected participants.

However, other studies found higher prevalences compared with our study. A pre-HAART era Botswana study found a higher HIV-HBV co-infection of $10.6 \%$ among urban participants. ${ }^{9} \mathrm{~A}$ systematic review estimated that the prevalence of HIV-HBV coinfection in sub-Saharan Africa was $14.9 \%{ }^{6}$

Studies reporting higher prevalences seemed to be mostly those conducted prior to the inception of the various national HAART programmes, as the majority of study participants could have had severe immunosuppression, which predisposed them to HBV reactivations and re-infections. ${ }^{9,14,15}$ The study settings could also have been a contributing factor to higher prevalence rates, especially studies conducted among inpatients in tertiary teaching hospitals. ${ }^{16,17}$

The 30-39 age group had the highest number of participants $(46 / 118,39.0 \%)$, whereas the $20-29$ age group accounted for half of the co-infected participants in our study. The high number of participants in the 30-39 age group coincides with the age group that bears the highest HIV prevalence in Botswana. ${ }^{18}$

Our study used HBsAg testing as the sole marker to identify hepatitis $B$ infection among participants. Additional tests such as antibody to hepatitis B core (Immunoglobulin [lg] M, G fractions), antibody to hepatitis B core lgG fraction, and polymerase chain reaction for hepatitis $B$ virus deoxyribonucleic acid were not part of the study due to the financial constraints. Therefore we could not determine whether the hepatitis B infection was acute or chronic, neither could we diagnose occult hepatitis. The prevalence found in our study may marginally underestimate the magnitude of HIV-HBV co-infection as it excluded occult hepatitis, which is a presence of HBV infection with undetectable hepatitis B surface antigen ( $\mathrm{HBsAg}$ ).

On the other hand, it was not part of this study to verify the persistence of HBsAg over six months; we made the assumption that $\mathrm{HBsAg}$ reactivity represented chronic infection. This could have led to an over-estimation of the co-infection prevalence in our study as some of our patients might have had acute infection that cleared within six months.

The adverse effects of HIV-HBV co-infection status warrant the investigation of various intervention measures in the context of Botswana. Currently, the WHO and the Southern African HIV Clinicians Society recommend HBV screening prior to HAART initiation and since 2005 this is no longer part of routine practice in Botswana. ${ }^{19,20}$

Table 2: Health district distribution of participants and HIV-HBV co-infected participants in the Mahalapye, Ngami and Serowe health districts; August to October 2015

\begin{tabular}{lccccc}
\hline & Number of HAART eligible participants $(\boldsymbol{n}=\mathbf{1 1 8})$ & & Number of HIV-HBV co-infected participants $(\boldsymbol{n}=\mathbf{6})$ \\
\cline { 2 - 3 } \cline { 5 - 6 } Health district & Males $(\boldsymbol{n}=\mathbf{3 8})$ & Females $(\boldsymbol{n}=\mathbf{8 0})$ & 26 & Males $(\boldsymbol{n}=\mathbf{2})$ & Females $(\boldsymbol{n}=\mathbf{4})$ \\
\hline Mahalapye & 16 & 30 & 0 & 1 \\
Ngami & 11 & 24 & 2 & 2 \\
Serowe & 11 & 80 & 2 & 1 \\
Total & 38 & & 0 & 4 \\
\hline
\end{tabular}


Even though HBsAg serological testing is both financially and technically challenging, it is not always sufficient to detect HBV infection in the setting of HIV co-infection as it excludes the diagnosis of occult hepatitis. Therefore the endorsement of two WHO pre-approved HBsAg rapid test kits (RDT) in Botswana, and consideration of their simultaneous use per patient for preHAART HBV screening, could benefit most of the HIV infected patients at a modest cost. Additionally, the use of RDTs could allow for point-of-care and out-of-health-facility HBV screening.

The prevalence of occult hepatitis amongst the general population is considered to be low; however, the findings of a recent South African study are suggestive of a possible association between HIV co-infection and high occult hepatitis rates, hence the need to maintain a high index of suspicion when dealing with 'unexplained' post-HAART initiation hepatic flares. ${ }^{21}$

The 'Treat All' approach for HIV management was launched in Botswana in June 2016 and uses the (TDF) and (FTC) backbone HAART regimen with dolutegravir (DTG) as the third drug. ${ }^{22}$ This regimen will control undiagnosed $\mathrm{HBV}$ infection in our setting as pre-HAART HBV screening is not done routinely. More robust routine HIV testing and HBV screening efforts need to be implemented so as to achieve the benefits of the 'treat all' approach. Such benefits include controlling HBV infection among co-infected patients and reducing MTCT of HBV among HIV co-infected pregnant women, over and above reducing new HIV infections and curtailing the incidence of opportunistic infections. The prevention of HBV infection in Botswana is currently based on routine vaccination schedules of newborns and health workers, implementation of universal precautions, promotion of condom use and health education to avoid body fluids and blood contact. Some patients with chronic hepatitis B infection may be treated with two antiviral drugs such as TDF, FTC and 3TC alone in the absence of HIV co-infection or along with DTG in the presence of HIV co-infection.

\section{Conclusion}

Our study finding was that $5.1 \%$ of HAART-eligible adult patients had HBV co-infection in the study sites. The co-infection rate was not uniform across the three selected rural sites. The 20-29 age group accounted for half of the HIV-HBV co-infected patients. Further studies covering more rural areas in Botswana could provide a more representative result that could facilitate adoption of a public health approach towards viral hepatitis. Such an approach could assist Botswana to achieve the 2030 Sustainable Development Goals (SDG 3.3$)^{23}$ to eliminate viral hepatitis as a major public health threat.

Acknowledgement - This study was conducted in partial fulfilment of the requirements for the award of the Master of Medicine (Family Medicine) degree at the University of Botswana. The authors acknowledge the valuable contributions of the Mahalapye, Ngami and Serowe Health Districts HAART clinics' staff, as well as those made by the study participants.

Conflict of interest - The authors declare that they had no financial or personal relationships which may have inappropriately influenced them in writing this article.

\section{References}

1. WHO. Global Health Sector Strategies on HIV, viral hepatitis and Sexually Transmitted Infections (STIs) 2016-2021. Global Health Sector Strategies 2016-2021 (GHSS). Briefing Note: October 2015 [cited Oct 25, 2016]; 2015. p. 1-9. Available from http://www.who.int/ hiv/strategy2016-2021/GHSS_Briefing_Note_Final_October2015.pdf
2. UNAIDS. AIDS by the numbers - factsheet [cited 25 Oct, 2016]. 2015. p. 1-11. Available from: www.unaids.org/en/resources/ documents/2015/AIDS_by_the_numbers_2015

3. WHO. Global Health Sector strategy on viral hepatitis 2016-2021. Towards ending viral hepatitis [cited 25 Oct, 2016]. 2015 Oct. p. 1-56. Available from: app.who.int/iris/bitstream/10665/246177/1/WHOHIV-2016.06-eng.pdf.

4. CDC.Viral hepatitis - CDC recommendations for specific populations and settings. Division of viral hepatitis and National Center for HIV/ AIDS, Viral Hepatitis, STD and TB prevention [cited 25 Oct, 2016]. 2014. p. 1-2. Available from: www.cdc.gov/hepatitis/populations/ pdfs/hivandhep-factsheet.pdf.

5. Marcellin P. Hepatitis B and hepatitis C in 2009. Liver Int. 2009;29(1):1-8. https://doi.org/10.1111/liv.2009.29.issue-s1

6. Barth RE. Hepatitis $B / C$ and HIV in sub-Saharan Africa: an association between highly prevalent infectious diseases. A systematic review and meta-analysis. Int J Infect Dis. 2010;14(12):e1024-31. https://doi.org/10.1016/j.ijid.2010.06.013

7. Hamers RL, Zaaijer HL, Wallis $\mathrm{CL}$, et al. HIV-HBV coinfection in southern Africa and the effect of lamivudine- versus tenofovir-containing CART on HBV outcomes. J Acquir Immune Defic Syndr. 2013;64(2):174-82. https://doi.org/10.1097/QAl.0b013e3182a60f7d

8. Matthews PC, Beloukas A, Malik A, et al. Prevalence and characteristics of hepatitis B virus (HBV) coinfection among HIV-positive women in South Africa and Botswana. PLoSONE. 2015;10(7):e0134037. https://doi.org/10.1371/journal.pone.0134037

9. Wester CW, Bussmann H, Moyo S, et al. Serological evidence of HIVassociated infection among HIV-1--infected adults in Botswana. Clin Infect Dis. 2006;43:1612-5. https://doi.org/10.1086/508865

10. Patel P, Davis S, Tolle MA, et al. Prevalence of hepatitis B and hepatitis $C$ coinfections in an adult HIV centre population in Gaborone, Botswana. Am J Trop Med Hyg. 2011;85(2):390-4. https://doi.org/10.4269/ajtmh.2011.10-0510

11. Hoffmann CJ, Thio CL. Clinical implications of HIV and hepatitis B co-infection in Asia and Africa. Lancet Infect Dis. 2007;7:402-9. https://doi.org/10.1016/S1473-3099(07)70135-4

12. Republic of Botswana. Botswana National HIV-AIDS Treatment Guidelines. Gaborone: Ministry of Health; 2012.

13. Alter MJ. Epidemiology of viral hepatitis and HIV co-infection. J Hepatol. 2006;44:S6-9. https://doi.org/10.1016/j.jhep.2005.11.004

14. Ahmed SD, Cuevas LE, Brabin BJ, et al. Seroprevalence of hepatitis B and C and HIV in Malawian pregnant women. J Infect. 1998;37(3):248-51. https://doi.org/10.1016/S0163-4453(98)91983-1

15. Burnett RJ, Francois G, Kew MC, et al. Hepatitis B virus and human immunodeficiency virus co-infection in sub-Saharan Africa: a call for further investigation. Liver Int. 2005;25(2):201-13. https://doi.org/10.1111/liv.2005.25.issue-2

16. Nakwagala FN, Kagimu MM. Hepatitis B virus and HIV infections among patients in Mulago Hospital. East Afr Med J. 2002;79(2):68-72.

17. Nyirenda $M$, Beadsworth MBJ, Stephany $P$, et al. Prevalence of infection with hepatitis $B$ and $C$ virus and coinfection with HIV in medical inpatients in Malawi. J Infect. 2008;57:72-7. https://doi.org/10.1016/j.jinf.2008.05.004

18. Republic of Botswana. Progress report on the National response to the 2011 declaration of commitments on HIV and AIDS. Gaborone: National Aids Coordinating Agency; 2014.

19. WHO Guidelines. Management of Hepatitis B and HIV coinfection. Copenhagen: Clinical protocol for the WHO European region; 2011.

20. Southern African HIV Clinicians Society guidelines. Management of HIV-Hepatitis B co-infection; Durbanville. 2011 Mar.

21. Mayaphi SH, Roussow TM, Masemola DP, et al. HBV/HIV co-infection: the dynamics of HBV in South African patients with AIDS. S Afr Med J. 2012;102(3):157-62. https://doi.org/10.7196/SAMJ.4944

22. Republic of Botswana. Handbook of the Botswana Integrated HIV Clinical care guidelines. Gaborone: Ministry of Health; 2016. p. 1-44.

23. UN. General Assembly. Resolution adopted by the General Assembly. Transforming our world: The 2030 Agenda for sustainable development. New York (NY). 2015 Sep. 